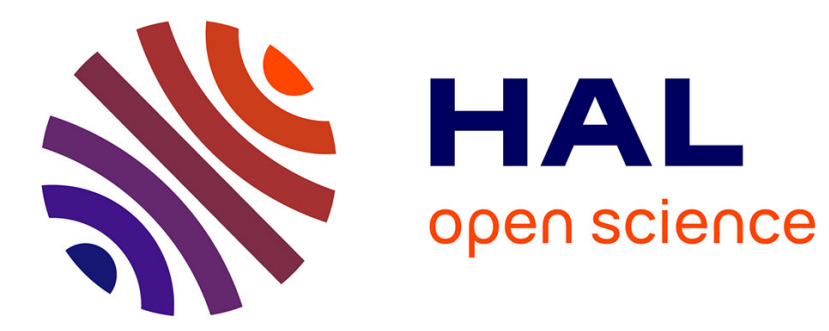

\title{
The impact of stretching-enhanced mixing and coalescence on reactivity in mixing-limited reactive flows
}

Sabyasachi Sen, Prajwal Singh, Joris Heyman, Tanguy Le Borgne, Aditya Bandopadhyay

\section{- To cite this version:}

Sabyasachi Sen, Prajwal Singh, Joris Heyman, Tanguy Le Borgne, Aditya Bandopadhyay. The impact of stretching-enhanced mixing and coalescence on reactivity in mixing-limited reactive flows. Physics of Fluids, 2020, 32 (10), pp.106602. 10.1063/5.0022798 . insu-03007232v2

\section{HAL Id: insu-03007232}

\section{https://hal-insu.archives-ouvertes.fr/insu-03007232v2}

Submitted on 17 Dec 2020

HAL is a multi-disciplinary open access archive for the deposit and dissemination of scientific research documents, whether they are published or not. The documents may come from teaching and research institutions in France or abroad, or from public or private research centers.
L'archive ouverte pluridisciplinaire HAL, est destinée au dépôt et à la diffusion de documents scientifiques de niveau recherche, publiés ou non, émanant des établissements d'enseignement et de recherche français ou étrangers, des laboratoires publics ou privés. 


\section{The impact of stretching-enhanced mixing and coalescence on reactivity in mixing- limited reactive flows}

Cite as: Phys. Fluids 32, 106602 (2020); https://doi.org/10.1063/5.0022798

Submitted: 23 July 2020 . Accepted: 15 September 2020 . Published Online: 06 October 2020

Sabyasachi Sen (D), Prajwal Singh (D), Joris Heyman (D), Tanguy Le Borgne, and Aditya Bandopadhyay (i)

\section{COLLECTIONS}

F This paper was selected as Featured 


\title{
The impact of stretching-enhanced mixing and coalescence on reactivity in mixing-limited reactive flows
}

\author{
Cite as: Phys. Fluids 32, 106602 (2020); doi: 10.1063/5.0022798 \\ Submitted: 23 July 2020 - Accepted: 15 September 2020 • \\ Published Online: 6 October 2020
}

Sabyasachi Sen, ${ }^{1}$ (D) Prajwal Singh, ${ }^{1}$ (D) Joris Heyman, ${ }^{2}$ (D) Tanguy Le Borgne, ${ }^{2}$ and Aditya Bandopadhyay ${ }^{1, a)}$ (D)

AFFILIATIONS

${ }^{1}$ Department of Mechanical Engineering, Indian Institute of Technology Kharagpur, Kharagpur, West Bengal 721302, India

${ }^{2}$ Céosciences Rennes, Université de Rennes 1, CNRS, UMR 6118, 35000 Rennes, France

a) Author to whom correspondence should be addressed: aditya@mech.iitkgp.ac.in

\begin{abstract}
We analyze the dynamics of solute mixing and reaction in a mixing-limited reactive flow by considering the transport of a tracer in a linear shear flow and in a Rankine vortex. The action of a shear flow, in general, achieves stretching of fluid elements due to the heterogeneous nature of the flow. A vortex flow exhibits not only stretching but also folding of fluid elements in a way that brings adjacent fluid elements closer at every turn. A strong stretching along the tangential direction is accompanied by a concomitant thinning in the radial direction leading to a strong diffusive flux, which may cause the material from neighboring regions of the mixing interface to aggregate. Through a Lagrangian concentration evolution technique, the diffusive strip method, we obtain the concentration field and pinpoint the signature of coalescence of two neighboring concentration regions by analyzing the concentration distribution profiles. The role of substrate deformation on the reaction kinetics of a classical heterogeneous chemical reaction is also studied where we derive analytical expressions for the coupling between the rate of product formation and the Péclet number in different time limits. Finally, the impact of coalescence on reaction rates is studied for a Rankine vortex, a result that holds important implications for simple bimolecular reactions. This analysis is useful to understand scalar dispersion in vortical flow structures and the consequences of stretching-enhanced diffusion in mixing-limited reactive flows.
\end{abstract}

Published under license by AIP Publishing. https://doi.org/10.1063/5.0022798

\section{INTRODUCTION}

Modeling reactive transport involves a mathematical analysis of the evolution of the concentration of solutes that undergo a chemical reaction as they are transported in an underlying flow. Understanding the reactive dynamics is paramount in studies related to contaminant transport in the Earth's surface and subsurface flows, ${ }^{1,2}$ geological storage of carbon dioxide, ${ }^{3}$ fate of reactive solutes in oceans, clogging of geothermal installations and growth of biofilms, ${ }^{6,7}$ industrial mixers, ${ }^{8,9}$ and other chemical processes. ${ }^{10,11}$ The earliest studies on solute transport in porous media considered the evolution of conservative species that is typically modeled by the advectiondispersion equation. ${ }^{12-15}$ Due to the highly nonlinear nature of the equation governing the dynamics of solute transport, numerical approaches were generally used to obtain the solution. ${ }^{16-19}$
However, in the context of subsurface flows, this gives an incomplete picture of the reality since chemical reactions strongly influence the fate of solutes as they are transported with the flow. The effect of the chemical reaction is incorporated in transport problems by appropriately modifying the equations governing the transport of conservative solutes. This is done typically by adding (or subtracting) a source term, with the nature of the resulting differential equation depending strongly on the type of chemical reaction. ${ }^{20}$ Again, the nonlinear nature of the equations makes the problem analytically intractable and has resulted in a host of numerical codes to simulate reactive transport. ${ }^{21-24}$ In many situations, however, an analytical solution is desired in order to gain deeper physical insight into the solution and assess the influence of various transport and reaction parameters. This has led to the development of analytical solutions, most of which are based on the concept of a component, that is, a 
linear combination of the species that remains conserved during the transport process. ${ }^{25}$ The idea of components has simplified the problem of solute transport by enabling the decoupling of the transport equations from the chemical relation equations, where the chemical relations were either of the chemical equilibrium type or of the chemical kinetic type. ${ }^{26-28}$

From a groundwater system perspective, there has been an enormous amount of academic interest over the decades in this area due to the importance of simulating the extent of damage due to pollutants at uncontaminated sites, assuming the geomorphology is known, and to predict the effectiveness of remedial measures at contaminated sites. ${ }^{29-32}$ Due to large transport times in groundwater flows, the chemical reactions during solute transport in such systems are assumed to be of the "sufficiently fast" reversible type such that locally, chemical equilibrium equations are valid everywhere in the domain. ${ }^{27}$ The assumption of fast reactions implies that the characteristic time scale for the reaction is several orders of magnitude smaller than that of any transport process which alters the concentration of the solute. Such a system is called mixing-limited since the driving force for chemical reactions is the disequilibrium induced due to the simultaneous processes of advection and diffusion, that is, mixing.

The influence of mixing on reaction kinetics has been studied for the mixing of waters with different chemical composition. ${ }^{33-35}$ Recently, emphasis has been put on understanding the role of fluid deformation on mixing rates and its consequences for reactivity. ${ }^{36-40}$ The stretching of fluid elements by a heterogeneous flow field is known to exert a dominant control on mixing rates of scalar fields. ${ }^{41-43}$ While the mechanisms leading to the elongation of material lines are well understood, predicting mixing rates and understanding their influence on reaction kinetics remains a challenge particularly when there is a reconnection (or aggregation) between several parts of the mixing interface, leading, at large mixing time, to a so-called coalescence regime. ${ }^{4-49}$ Understanding the dynamics of this coalescence regime becomes important in flows of geophysical relevance since coalescence destroys concentration gradients, thereby influencing mixing rates. ${ }^{50,51}$ On the other hand, sharp gradients in concentration form fronts that act as hotspots for chemical reactions. ${ }^{52-54}$

In this work, we analyze the effect of stretching-enhanced mixing and coalescence dynamics on reactivity through scalar transport in a linear shear flow and in a Rankine vortex. To solve the transport problem, we propose a Lagrangian method that extends the Ranz stretch framework to model high Péclet number reactive flows. ${ }^{55,56}$ We use this Lagrangian method to reconstruct the scalar concentration fields and to compute the evolution of the distribution of concentration levels in time. The analytical solution for the solute concentration is substituted in the speciation equation to obtain closed form solutions for the upscaled reactivity and mass of precipitate as a function of time. Finally, we compare the integrated reactivity obtained from the independent lamellar model and that from a numerical reconstruction of the spatial concentration distribution to emphasize the impact of coalescence on reaction kinetics. These analytical results highlight the power of our computational model as they offer valuable insight into the interplay between reactive transport parameters and rates of mixing and the reaction in different time limits of a given stirring protocol. These insights are difficult to glean from Eulerian simulations where analytical solutions are found to be lacking. Moreover, the time taken for computation in the Lagrangian method is independent of the Péclet number because advection dominated flows can be modeled to be an advection step on to which diffusion occurs. These points make our formulation an excellent alternative to Eulerian methods for solving reactive transport problems, especially in the high Péclet number regime.

\section{NUMERICAL METHODS}

\section{A. Mathematical formulation}

In this work, we analyze a chemical system consisting of a single heterogeneous bimolecular reaction where two reactive species are in equilibrium with a precipitate $\left[A_{(a q .)}+B_{(a q .)} \rightleftharpoons C_{(s)}\right]$. We assume a simplified setting where the precipitate does not interact with the background flow and leaves transport properties in the domain unchanged. The reactants are denoted by $A$ and $B$, and the solid product is denoted by $C$; their dimensional concentrations are $a, b$, and $c$, respectively. Expressing the reactivity as $r$ and assuming a homogeneous, isotropic diffusion coefficient $D$, the transport equations are given by

$$
\begin{aligned}
\frac{\partial a}{\partial t}+\mathbf{v} \cdot \nabla a & =D \nabla^{2} a-r \\
\frac{\partial b}{\partial t}+\mathbf{v} \cdot \nabla b & =D \nabla^{2} b-r, \\
\frac{\partial c}{\partial t} & =r .
\end{aligned}
$$

We can justify the assumption of a homogeneous, isotropic diffusion coefficient $D$ by considering the dedolomitization reaction where the chemical system consists of four equilibrium reactions. ${ }^{27}$ Let us look specifically at the sub-system consisting of the reaction where a calcium cation reacts with a carbonate anion to give a calcium carbonate precipitate since it resembles our system. Results from the capillary tube method ${ }^{57}$ and molecular dynamics simulations ${ }^{58}$ show that all three species in the chemical reaction considered have diffusion coefficients in sea water of the order of $10^{-9} \mathrm{~m}^{2} / \mathrm{s}$. Since linear shear flows are often used to model saltwater intrusion in freshwater, the assumption of equal diffusion coefficients as experimentally found in seawater is a good assumption to use in our formulation. We use the idea of components, which are linear combinations of chemical species that are conserved during transport, to reduce the mathematical complexity of the transport problem. ${ }^{25,59,60}$ Using the framework introduced in Ref. 27, we deduce that our chemical system contains only one component, $q=a-b$, such that the transport problem reduces to the conventional advection-diffusion equation in $q$,

$$
\frac{\partial q}{\partial t}+\mathbf{v} \cdot \nabla q=D \nabla^{2} q
$$

Under the assumption of a mixing-limited reaction, the chemical relation equation takes the form of the chemical equilibrium equation $a b=K_{e q}$, where we assume that the stoichiometric coefficients are unitary and $K_{e q}$ is a constant (not a function of either time or position in space). The equilibrium equation can be solved to yield 
the solute concentrations in terms of the component concentration as follows:

$$
a=\frac{q+\sqrt{q^{2}+4 K_{e q}}}{2}, \quad b=\frac{-q+\sqrt{q^{2}+4 K_{e q}}}{2},
$$

where the form of Eq. (3) implies the inherent coupling between transport and speciation and requires us to solve for Eq. (2). Eulerian methods are useful numerical tools to model the advectiondiffusion process but require great computational power to completely resolve the concentration field for high Péclet number flows. Due to this shortcoming, efforts have been made to develop alternative modeling techniques based on the Lagrangian framework. In our study, we integrate the Lagrangian concentration evolution technique called "the diffusive strip method" with particle tracking routines to analyze the evolution of a passive scalar. In the first step of the algorithm, scalar strips are advected in a velocity field given a priori, and their positions are computed kinematically by solving the equation $\frac{d \mathbf{x}_{\mathbf{i}}}{d t}=\mathbf{v}_{\mathbf{i}}\left(\mathbf{x}_{\mathbf{i}}\right)$ using an explicit Runge-Kutta method. The transport problem is made analytically tractable by applying the lamellar representation of mixing where we shift to a frame of reference fixed on the scalar support. ${ }^{55,56}$ The passive scalar is shown in its initial configuration, as a straight material line, represented by a finite number of points and in a deformed state at a later point in time in Fig. 1, where for strip $i, \sigma_{\mathbf{i}}$ and $n_{i}$ denote the local longitudinal direction and local transverse direction, respectively. The figure also shows other physical quantities that are fundamental to our formulation. In the initial state, we have shown the initial striation thickness $s_{0}$ and initial distance $\Delta x_{i}^{0}$ between points $\mathbf{x}_{\mathbf{i}}$ and $\mathbf{x}_{\mathbf{i}+\mathbf{1}}$, both of which are uniform throughout the material line. However, due to the heterogeneity in the underlying flow field, the strips are deformed non-uniformly leading to a striation thickness, $s_{i}$, and distance between consecutive points $\Delta x_{i}$, both of which vary from one

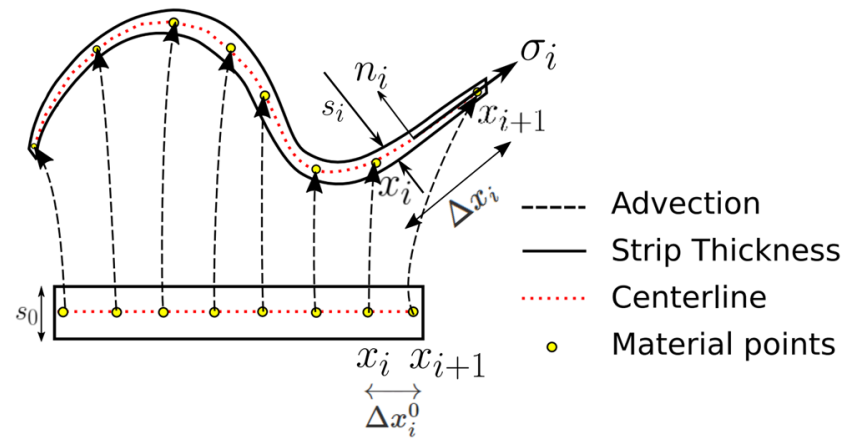

FIG. 1. Schematic of the tracer representation using a finite number of points at the initial time instant shown as a straight material line and at a later time instant in a deformed configuration. In the initial configuration, the material line has a uniform thickness, $s_{0}$, and a uniform gap between consecutive points $x_{i}$ and $x_{i+1}$, denoted by $\Delta x_{i}^{0}$ where the superscript 0 corresponds to the value at the initial time instant and $i$ is the index of the material point. In the deformed configuration, the material line has a non-uniform striation thickness and a non-uniform distance between consecutive tracers, that is, between $x_{i}$ and $x_{i+1}$ being $s_{i}$ and $\Delta x_{i}$, respectively. The local coordinate system corresponding to the strip of scalar between $x_{i}$ and $x_{i+1}$ is also shown with the direction along the curve being denoted by $\sigma_{i}$ and that perpendicular to the curve being denoted by $n_{i}$. strip to another in the same material line. The quantities are related to each other by the conservation of areas $s_{i}=\left(s_{0} \Delta x_{i}^{0}\right) / \Delta x_{i}$, which is a useful expression in the derivation of the velocity field in a frame of reference fixed on the material line. This transformation of the frame of reference allows us to account for diffusion, independent of advection, by inserting diffusive material segments along the strip. We represent the velocity field in the local coordinate system as a first-order Taylor expansion of the flow around $\mathbf{x}_{\mathbf{i}}$,

$$
\begin{gathered}
v_{\sigma} \mid \mathbf{x}_{\mathbf{i}} \approx \frac{\partial v_{\sigma_{i}}}{\partial \sigma_{i}} \sigma_{i}+\frac{\partial v_{\sigma_{i}}}{\partial n_{i}} n_{i}=-\frac{\sigma_{i}}{s_{i}} \frac{d s_{i}}{d t}+\frac{\partial v_{\sigma_{i}}}{\partial n_{i}} n_{i}, \\
v_{n} \mid \mathbf{x}_{\mathbf{i}} \approx \frac{\partial v_{n_{i}}}{\partial n_{i}} n_{i}=\frac{n_{i}}{s_{i}} \frac{d s_{i}}{d t} .
\end{gathered}
$$

The expression above, for $d \sigma_{i} / d t$ or equivalently, $v_{\sigma} \mid \mathbf{x}_{\mathbf{i}}$, is simplified using the conservation of areas to obtain $\frac{d}{d t}\left(\ln s_{i}\right)=-\frac{1}{\sigma_{i}} \frac{d}{d t} \sigma_{i}$. In this model, the scalar is assumed to undergo a series of stretching and folding operations, thereby organizing itself into an elongated filament-like structure. ${ }^{61-63}$ The deformation of the strip of scalar is such that its characteristic length scale in the local normal coordinate is much smaller than that along the material line, which allows us to neglect concentration gradients along the strip in comparison to those normal to the strip. Hence, it is mostly the diffusion-reaction process occurring in the direction normal to the strip that contributes to mass transfer, effectively decoupling the transport due to advection only and diffusion-reaction (only normal to the orientation of the strip). Considering a solitary strip in the material line and dropping the subscript index $i$, this simplifying assumption modifies the governing differential equations in the frame of reference attached to the tracer to

$$
\begin{aligned}
& \frac{\partial a}{\partial t}+\frac{n}{s} \frac{d s}{d t} \frac{\partial a}{\partial n}=D \frac{\partial^{2} a}{\partial n^{2}}-r, \\
& \frac{\partial b}{\partial t}+\frac{n}{s} \frac{d s}{d t} \frac{\partial b}{\partial n}=D \frac{\partial^{2} b}{\partial n^{2}}-r .
\end{aligned}
$$

However, the implicit coupling between substrate deformation and the extent of diffusion and/or reaction is obtained by transforming the equations to the warped coordinates: ${ }^{55} \tilde{n}=n / s$ and $\tau(t)=\frac{D}{s_{0}^{2}} \int_{0}^{t} \rho\left(t^{\prime}\right)^{2} d t^{\prime}$, where $\rho=s_{0} / s$. Using the chain rule for differentiation, the terms in Eq. (5) are transformed as follows:

$$
\begin{gathered}
\frac{\partial a}{\partial t}=\frac{\partial a}{\partial \tau} \frac{\partial \tau}{\partial t}+\frac{\partial a}{\partial \tilde{n}} \frac{\partial \tilde{n}}{\partial t}=\frac{D}{s^{2}} \frac{\partial a}{\partial \tau}-\frac{\tilde{n}}{s} \frac{d s}{d t} \frac{\partial a}{\partial \tilde{n}}, \\
\frac{n}{s} \frac{d s}{d t} \frac{\partial a}{\partial n}=\frac{\tilde{n}}{s} \frac{d s}{d t} \frac{\partial a}{\partial \tilde{n}}, \\
D \frac{\partial^{2} a}{\partial n^{2}}=\frac{D}{s^{2}} \frac{\partial^{2} a}{\partial \tilde{n}^{2}} .
\end{gathered}
$$

Using similar expressions for the reactant concentration $b$ and substituting (6) in (5), we obtain the simplified governing differential equations in the warped coordinates as

$$
\begin{aligned}
& \frac{\partial a}{\partial \tau}=\frac{\partial^{2} a}{\partial \tilde{n}^{2}}-\frac{s^{2}}{D} r, \\
& \frac{\partial b}{\partial \tau}=\frac{\partial^{2} b}{\partial \tilde{n}^{2}}-\frac{s^{2}}{D} r,
\end{aligned}
$$


where $r$ is the local reactivity or the source term corresponding to a solitary strip in the material line. This problem can be reduced to the one-dimensional diffusion equation for the component $q$ in the warped set of coordinates by subtracting (7b) from (7a) such that the governing equation finally reduces to

$$
\frac{\partial q}{\partial \tau}=\frac{\partial^{2} q}{\partial \tilde{n}^{2}}
$$

Assuming an initial distribution of $a$ and $b$ such that $q=a-b$ initially has a Gaussian distribution given by $e^{-\tilde{n}^{2}}$, we get the classical solution for a diffusion equation in an infinite domain,

$$
q(\tilde{n}, t)=\frac{1}{\sqrt{1+4 \tau(t)}} \exp \left(\frac{-\tilde{n}^{2}}{1+4 \tau(t)}\right) .
$$

Equation (9) indicates that all we need to compute during the advection problem in the diffusive strip method are the positions $\boldsymbol{x}_{i}$, warped times $\tau_{i}$, and lamella thicknesses $s_{i}$ of the strips in order to reconstruct the spatial distribution of the scalar. This is carried out numerically by adding Gaussian ellipses that are centered at the midpoint of the segment connecting consecutive points on the material strip,

$$
q(\mathbf{x})=\sum_{i} \frac{1 / 1.7726}{\sqrt{1+4 \tau_{i}(t)}} \exp \left(-\frac{\left[\left(\mathbf{x}-\mathbf{x}_{\mathbf{i}}\right) \cdot \sigma_{\mathbf{i}}\right]^{2}}{\Delta x_{i}^{2}}-\frac{\left[\left(\mathbf{x}-\mathbf{x}_{\mathbf{i}}\right) \cdot \mathbf{n}_{\mathbf{i}}\right]^{2}}{s_{i}^{2}\left(1+4 \tau_{i}(t)\right)}\right)
$$

where $\sigma_{\mathbf{i}}$ and $\mathbf{n}_{\mathbf{i}}$ are the unit vectors along and perpendicular to strip $i$ in the material line (see Fig. 1).

We may now obtain the mass of precipitate during such a flow. An explicit expression for the reactivity due to a solitary strip is obtained by substituting the solution for $b$ from Eq. (3) into the corresponding transport equation $(7 \mathrm{~b})$,

$$
\frac{\partial c}{\partial \tau}=\frac{s^{2}}{D} r=\frac{2 K_{e q}}{\left(q^{2}+4 K_{e q}\right)^{3 / 2}}\left(\frac{\partial q}{\partial \tilde{n}}\right)^{2} .
$$

The two soluble species, when in equilibrium, yield an insoluble static species whose rate of formation per unit area, in the limit of $K_{e q} \gg q^{2}$, is governed by

$$
\frac{\partial c}{\partial \tau}=\frac{s^{2}}{D} r=\frac{1}{4 \sqrt{K_{e q}}}\left(\frac{\partial q}{\partial \tilde{n}}\right)^{2} .
$$

The dimensional version of this result can be obtained by substituting $n=s \tilde{n}$ and by defining $D=U L / \mathrm{Pe} \Rightarrow D \approx 1 / \mathrm{Pe}$ [assume $U, L \sim \mathcal{O}(1)]$

$$
r=\frac{1}{4 \mathrm{Pe} \sqrt{K_{e q}}}\left(\frac{\partial q}{\partial n}\right)^{2}
$$

This is a key result since it highlights the explicit dependence of the reactivity on the mixing rate, quantified by $(\partial q / \partial n)^{2} / \mathrm{Pe}$, an expression which is consistent with the idea of a dilution index. ${ }^{64}$ In order to obtain the mass of precipitate as a function of time, due to the reaction with a solitary strip, we integrate Eq. (12) with respect to the spatial coordinates and the warped time to obtain

$$
\begin{aligned}
m_{c} & =\int_{0}^{\tau} \int_{l} \int_{-\infty}^{\infty} \frac{\partial c}{\partial \tau} d n d l d \tau \\
& =s_{0} l_{0} \int_{0}^{\tau} \int_{-\infty}^{\infty} \frac{\tilde{n}^{2}}{\sqrt{K_{e q}}(1+4 \tau)^{3}} \exp \left(\frac{-2 \tilde{n}^{2}}{1+4 \tau}\right) d \tilde{n} d \tau,
\end{aligned}
$$

where $l_{0}$ and $l$ denote the length of the solitary strip at the initial time and at an arbitrary time, respectively (note that $l_{0}$ represents the same quantity as $\Delta x_{i}^{0}$, but in this part of the mathematical formulation, we do not use the subscript index $i$ because we are dealing with a solitary strip and not an arbitrary number of strips). Upon integration, we finally obtain

$$
m_{c}=\frac{s_{0} l_{0}}{16} \sqrt{\frac{2 \pi}{K_{e q}}}\left(1-\frac{1}{\sqrt{1+4 \tau}}\right) .
$$

The lamellar mixing model is valid in the regime of no coalescence and assumes that each strip evolves independent of all others in the flow field, allowing us to upscale to the total mass of precipitate formed in the entire domain by adding the contribution from each strip $m_{c, \text { total }}=\sum_{i} m_{c}$.

\section{B. Reaction kinetics in a linear shear flow}

In order to test the validity of the diffusive strip method as a viable alternative to Eulerian methods, we consider the prototypical case of a linear shear flow to show the excellent agreement between the results obtained from simulations carried out in the two different modeling frameworks. The flow field $\vec{V}=u \hat{i}+v \hat{j}$ is given by

$$
(u, v)=(y, 0) .
$$

A strip of scalar of unit length and characteristic thickness $s_{0}=0.1$ units is initially placed along the $\mathrm{y}$ axis at $\mathrm{x}=0$ and is sheared by this flow field. We reconstruct the concentration field and derive the PDF of concentration using both the Eulerian and Lagrangian schemes. In the PDF, the concentration $c$ has been normalized by the maximum concentration $c_{0}$, which in turn is assumed throughout our study to be 1 . A very good match between the results from the two modeling methods is observed in Fig. 2 for a range of Péclet numbers. A careful comparison between the PDFs obtained from the Lagrangian and Eulerian simulations reveals, however, that the Eulerian PDFs show a slightly greater decay in concentration than that shown in the results obtained from the diffusive strip method. This additional decay is absent in the Lagrangian simulations precisely because here the advection and diffusion processes are decoupled. This decoupling causes the velocity field and its gradient to act only on points representing the centerline of the strip, and thus, the effect of the space-continuous velocity field on the mass comprising the entire material lamella is unaccounted for. This is why we observe uniform stretching normal to the strip in the images for the concentration field reconstructed from the Lagrangian simulations (see Fig. 7). This difference is due to the existence of a velocity gradient normal to the strip, i.e., $\frac{\partial u}{\partial n} \neq 0$, which causes differential stretching normal to the strip for the case of the Eulerian simulations. However, for most of the PDF, the two numerical schemes are in excellent agreement with one another since the differential stretching experienced locally normal to the strip, 


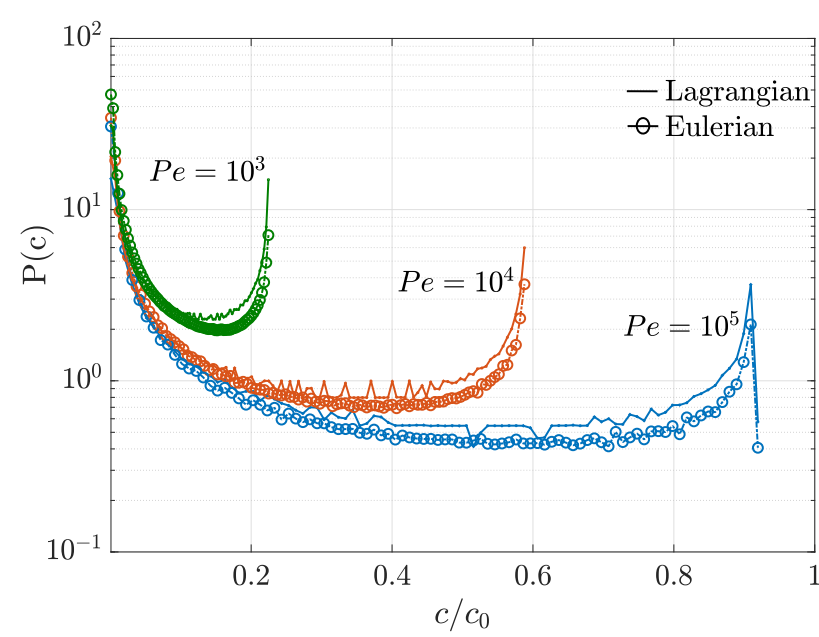

(a)

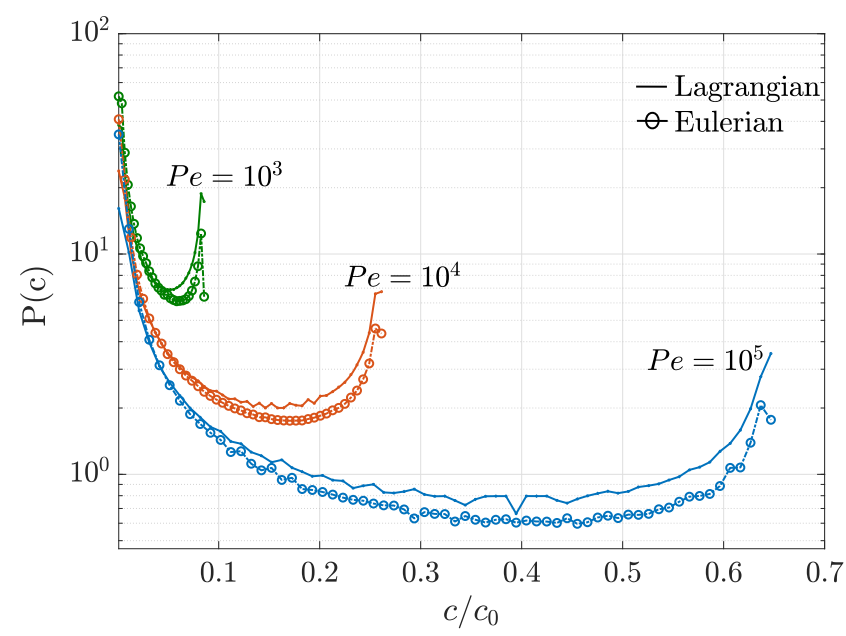

(b)

FIG. 2. An excellent agreement between the PDFs obtained from the Lagrangian and Eulerian simulations at (a) $t=5$ and $(b) t=10$ for a linear shear flow. The solid line corresponds to the Lagrangian simulation, whereas the dashed-dotted line with markers corresponds to the Eulerian simulation.

due to the non-zero contribution from $\frac{\partial u}{\partial n} \Delta n$, is negligible compared to the absolute velocity. This validation exercise establishes the much faster Lagrangian technique based on the diffusive strip method as a very good alternative to Eulerian methods, allowing us to proceed in our study of reactive mixing using the diffusive strip method.

Using the mathematical formulation derived in Sec. II A, we first demonstrate the reactive dynamics of a solitary strip being transported in a linear shear flow $(u, v)=(y, 0)$. Consider a material line of initial width $s_{0}=0.1$ placed along the y axis in $-0.5<y$ $<0.5$ at $x_{\text {pos }}=0$. The initial conditions for the species are chosen in such a manner that equilibrium is satisfied everywhere in the domain while yielding a Gaussian distribution for the conservative component, $q$.

In Fig. 3(a), we represent the evolution of the reactant masses toward equilibrium in the concentration space. At equilibrium, the concentration of both the species tends to the value of $\sqrt{K_{e q}}$. It is seen that the evolution follows the equilibrium curve $a b=K_{e q}$ [denoted by the line joining markers of a single color in Fig. 3(a)]. Disequilibrium conditions are constantly created due to the combined effects of advection and diffusion. A decrease in the concentration of $a$ results in traversal along the equilibrium curves. This traversal is equivalent to the two reactants attempting to reach the equilibrium condition of $a=b=\sqrt{K_{e q}}$ after which there are no more reactions in the system. It must be noted that as the system approaches the $a=b$ line, the rate of approach first increases and then decreases. In the initial stages, the fast rate of approach is due to the large driving force for reaction, which increases as long as concentration gradients increase and then decreases asymptotically to zero. The fact that the maximum reactivity does not correspond to the initial time but to a later time instant also holds interesting implications for the regions in which the maximum mass would be expected to be precipitated. Figure 3(b) represents the spatial evolution of the normalized concentrations of species $A$ and $B$ (relative to the equilibrium concentration $\sqrt{K_{e q}}$ ), and the upper set of curves represents species $A$ along the normal coordinate $n$ at time intervals of $\Delta t=1$. The concentration profiles of the reactants are distributed about the eventual equilibrium concentration $\sqrt{K_{e q}}$ in such a manner that the product is always constrained to $a b=K_{e q}$. Such an evolution of the concentration profile has also been reported by De Simoni et al. ${ }^{2}$

As time progresses, we see that instead of concentration gradients attenuating by diffusing immediately from the initial profile, there is first an increase in the normal concentration gradient. This occurs since, in the initial stages of the deformation protocol, shear is the dominant mechanism governing the evolution of the scalar, thereby causing a net reduction in strip width. The thinning continues until the rate of compression of the strip is equilibrated by the rate of increase in the thickness due to diffusion $\frac{s_{0}}{\nabla v t} \sim \sqrt{D t}$ (here, $\nabla v$ is the stretching rate; see Fig. 6). The time at which this event occurs is known as the mixing time $t_{m i x}$ and this is also the time at which concentration gradients are at a maximum. Beyond the mixing time, the width of the strip increases [Fig. 6(b)] and as a result, attenuates concentration gradients. It was shown through Eq. (13) how the reactivity is related to the quantity of mixing of the conservative species. $^{27}$ The important consequence of the fluid deformation dynamics on a reaction is that the heterogeneity in the flow leads to mixing and reactive behavior that is very different from what is observed in a homogeneous flow field. The compression locally normal to the strip drives an enhanced mass flux in that direction due to the increased gradient. This is clearly seen in Fig. 4 through the spatio-temporal evolution of the reactivity at different time instants. The form of Eq. (13) tells us that the reactivity is zero at the peak of the concentration (that is, at the center of the lamella) and is highest where the gradient in the concentration is the maximum. Due to the symmetry of the concentration profile about the centerline of the lamella, we have shown only one side of the distribution about the origin of the normal direction.

The concentration distributions depicted in Figs. 3(b) and 4 may now be used to study the reactivity integrated over the spatial 


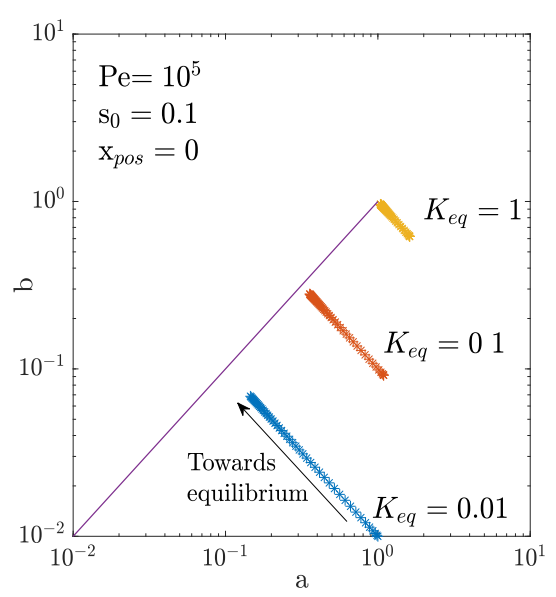

(a)

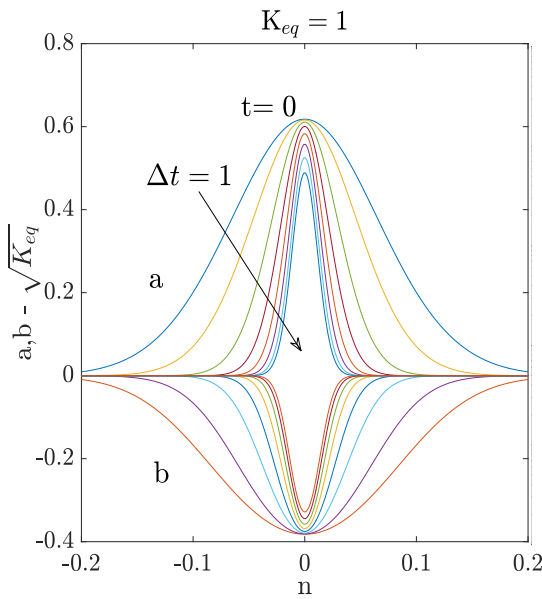

(b)

FIG. 3. (a) In a linear shear flow, we plot the evolution of the concentration of $A$ toward the global equilibrium state for different equilibrium constants. The markers are plotted from $t_{\text {start }}=0$ to $t_{\text {end }}=50$ at intervals of $\Delta t=1$. From the density of the markers, we can see that initially, the concentration a evolves at an increasing rate and then at a decreasing rate as it approaches the equilibrium line. This is an indicator of the driving force for the reaction, which is the reactivity. The faster increments in the concentration of a occur until a certain point in time when the concentration gradients are the highest, and in turn, the reactivity is also at its peak, beyond which the approach becomes slower. (b) The evolution of $\mathrm{a}$ and $\mathrm{b}$ toward the equilibrium concentration $\sqrt{K_{\text {eq }}}$ in a linear shear flow from $t=0$ (outer-most curves) to $t=7$ (inner-most curves) at intervals of $\Delta t=1$.

coordinates (in the direction normal to the strip and also along the strip). The fundamental premise of the lamellar approach is that the concentration variation (and consequently the reactivity) along the length of a solitary strip can be neglected in comparison to that along

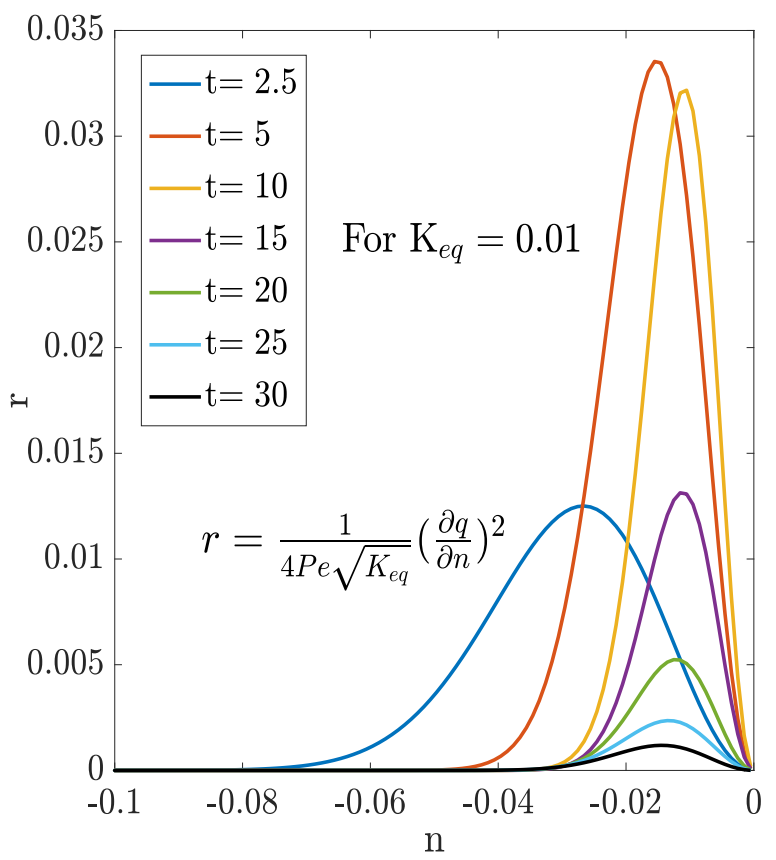

FIG. 4. The reactivity is plotted for a solitary strip in a linear shear flow along the local normal direction. It can be seen that the maximum reactivity is not reached at the initial injection time but at a later stage corresponding to the time and location when the concentration gradient is at a maximum. the transverse variation. This simplifies the spatial integration and results in the following solution for the integrated reactivity:

$$
R=\int_{l} \int_{-\infty}^{\infty} r d n d l=\frac{l}{4 \mathrm{Pe} \sqrt{K_{e q}}} \int_{-\infty}^{\infty}\left(\frac{\partial q}{\partial n}\right)^{2} d n
$$

Substituting the expression for $q$ given in Eq. (9) into the integral above yields

$$
R=\frac{l_{0} s_{0}}{\mathrm{Pes}^{2}} \frac{1}{8} \sqrt{\frac{2 \pi}{K_{e q}}} \frac{1}{(1+4 \tau)^{3 / 2}} \Longrightarrow \lim _{t \rightarrow 0} R \sim \frac{1}{\mathrm{Pe}} .
$$

This analysis is useful when it is important to have knowledge of the rate of formation and mass of product at any given instant of time. In Figs. 5(a) and 5(b), we depict the reactivity and temporal evolution of the mass of product, respectively. We observe a dependence of the initial time reactivity on the Péclet number as $\lim _{t \rightarrow 0} R$ $\sim 1 / \mathrm{Pe}$ and also observe that the rate at which the mass of precipitate is formed increases initially and then saturates. We may gain a better physical insight into the situation by observing that up until the mixing time, the reactivity increases since shear dominates diffusion, beyond which we observe a significant drop in the reactivity. This behavior of the reactivity is reflected in the variation of mass in time. At early times, the mass of product, which is obtained through integration in time of the reactivity term, is shown to vary as $m_{c} \sim t / \mathrm{Pe}$. This can be obtained from Eq. (15) by using the binomial expansion for $(1+4 \tau)^{-1 / 2}$ for small $\tau$ and by substituting $\tau=\frac{1}{\operatorname{Pes}_{0}^{2}}\left[t+\gamma^{2} t^{3} / 3\right]$. Beyond this stage, indicated accurately by the mixing time (black markers), there is a drastic decay in the reactivity and that is reflected in the saturation of the mass of the product formed. Importantly, we note that the limit of the total mass precipitated $\left(=\frac{\sqrt{2 \pi}}{16} s_{0} l_{\text {total }}\right.$, where $l_{\text {total }}$ is the initial length of the entire material line) at large times is independent of the Péclet number. Thus, the influence of the 


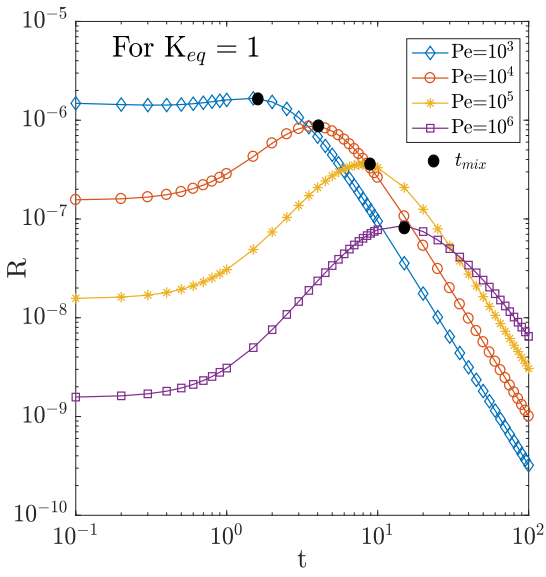

(a)

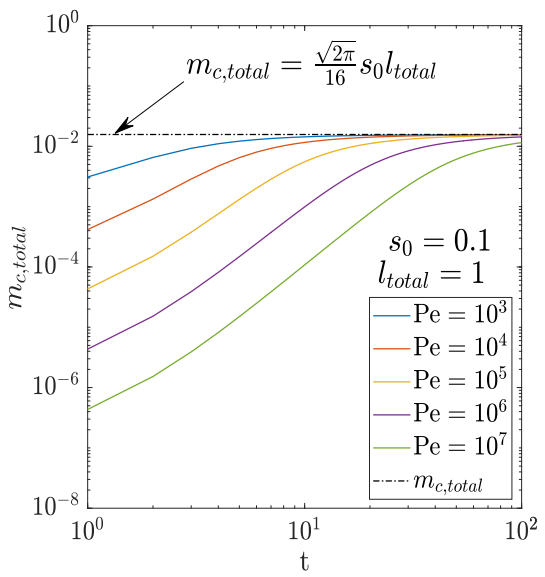

(b)
FIG. 5. (a) The integrated reactivity $R=\iint r d n d l$ is plotted against time for a solitary strip in a linear shear flow for a range of Péclet numbers (with $K_{e q}=1$ ). In each case, the reactivity in the initial stages is constant and is seen to scale as $1 / \mathrm{Pe}$, a result consistent with Eq. (18), and as expected from the lamellar description of mixing, decreases beyond the mixing time indicated by the black markers. (b) The total mass obtained in the domain is plotted for various Péclet numbers. All yield the same final mass but do so at different times. Low Péclet number flows reach the mixing time before that of high Péclet number flows and react faster to give the same final mass.
Péclet number is most prominently manifested at the initial times, as indicated by Eq. (18).

A scaling law is also derived relating the integrated reactivity with the Péclet number at long times beyond the mixing time. From Eq. (18) and by using $s=s_{0} / \sqrt{1+t^{2}}$ for a linear shear, we obtain

$$
R=\frac{s_{0} l_{0}}{8} \sqrt{\frac{2 \pi}{K_{e q}}} \frac{1}{\operatorname{Pes}_{0}^{2}} \frac{1+t^{2}}{\left[1+\frac{4}{\operatorname{Pes}_{0}^{2}}\left(t+\frac{t^{3}}{3}\right)\right]^{3 / 2}} .
$$

In the limit of $t \gg t_{m i x}$, this reduces to [Fig. 5(a)]

$$
R=\frac{s_{0}^{2} l_{0}}{64} \sqrt{\frac{2 \pi}{K_{e q}}} \mathrm{Pe}^{1 / 2} t^{-5 / 2} \sim \mathrm{Pe}^{1 / 2} t^{-5 / 2} .
$$

\section{RESULTS}

\section{A. Rankine vortex flow}

The Rankine vortex is a flow model with an inner circular zone comprising a forced vortex and an outer region characterized by a free vortex. The radial symmetry in the flow makes it appropriate to define the velocity field in the cylindrical polar coordinate system ( $r$, $\theta, \mathrm{z}$ ), where the $\mathrm{z}$ axis is the axis of symmetry and $\mathrm{r}$ and $\theta$ coordinates are in the plane of flow. The analytical expression for the Rankine vortex is as follows:

$$
\left(v_{r}, v_{\theta}, v_{z}\right)=\left\{\begin{array}{ll}
\left(0, c_{1} r, 0\right), & r \leq r_{c} \\
\left(0, \frac{c_{2}}{r}, 0\right), & r>r_{c}
\end{array},\right.
$$

in which case the velocity field attains a maximum at the characteristic distance of the vortex, $r_{c}$, where the flow field changes its nature from linear to hyperbolic. An important feature of this flow field, with consequences for turbulence, ${ }^{65}$ is a discontinuity in its vorticity, which is a constant $\left(=2 c_{1}\right)$ in the inner circular zone and a null vector in the outer region. Despite its simplicity, the Rankine vortex has frequently been used to model a generic phenomenon in the atmosphere, such as tornadoes and mesocyclones. ${ }^{66-68}$ At the same time, the outer free vortex region of the flow model, resembling a point vortex, is a common approximation to circulating regions of a stagnant fluid in porous media flows. ${ }^{69,70}$ Hence, the Rankine vortex becomes a good choice of a flow field to illustrate coalescence during scalar mixing and also explore the influence of coalescence dynamics on reactivity for a mixing-limited reactive flow.

In our numerical study, $c_{1}=2, c_{2}=0.5, r$ denotes the distance of the material lamella from the center of the vortex, and the critical radius where the flow field has a discontinuous slope is $r_{c}=0.5$. All values have been reported in a non-dimensional form by assuming the domain length scale to be $\mathcal{O}(1)$. A strip of scalar, initially placed along the $\mathrm{x}$ axis in the range $0.45<x<2.0$, is advected in the flow field of a Rankine vortex. We assume that in this flow field, the deformation of material lines is governed by a single stretching rate, similar to the case of a linear shear flow. We denote this stretching rate by $\nabla v$ and assume that it is equal to the velocity gradient in the radial direction. Shearing due to this radial velocity gradient increases the length of the strip linearly in time and causes a compression transverse to the strip due to the fact that the velocity field is solenoidal. Figure 6(a) shows this phenomenon yielding the following expression for the striation thickness $s$ :

$$
s=\frac{s_{0}}{\sqrt{1+\nabla v^{2} t^{2}}} \Longrightarrow s \approx \frac{s_{0}}{\nabla v t},
$$

once shear effects are significant. The thinning of the strip simultaneously increases the concentration gradient transverse to the strip, thereby, inducing a diffusive flux that increases the strip width as $\sqrt{t+t^{3}}$. The competing processes of striation thinning and diffusion broadening decrease the strip width until the mixing time, $t_{\text {mix }} \sim \nabla v^{-1} \mathrm{Pe}^{1 / 3}$, when the associated length scale is the well-known Batchelor scale $s_{B} \sim s_{0} \mathrm{Pe}^{-1 / 3}$; here, $\mathrm{Pe}=\left(s_{0}^{2} \nabla v / D\right)$. Beyond the mixing time, the rate of compression is not enough to balance the broadening due to diffusion and the strip width increases as $\sqrt{t / \mathrm{Pe}}$. By solving the advection problem and by keeping track of the striation thicknesses and warped times, we compute the width using $w_{i}=s_{i} \sqrt{1+4 \tau_{i}} .{ }^{56}$ We plot the evolution of this width, averaged over the strip, in time for a range of Péclet numbers in Fig. 6(b). In the calculation of the analytically averaged width, the value of $\nabla v$ is taken to be 0.85 , which is obtained by averaging $\nabla v=\left|\frac{\partial v}{\partial r}\right|$ over the radial 


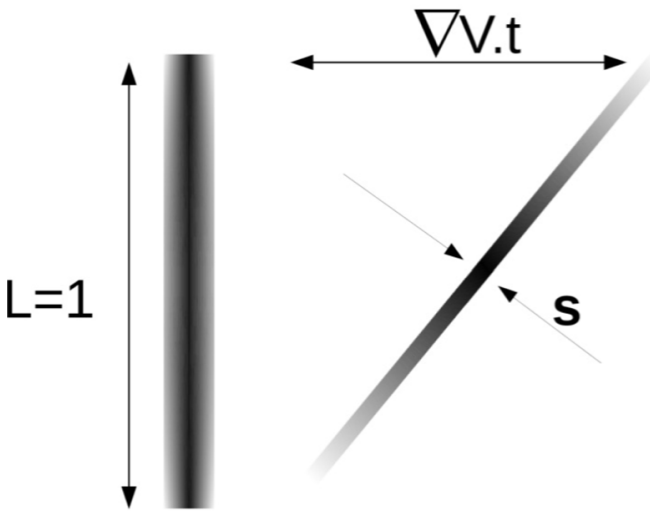

(a)

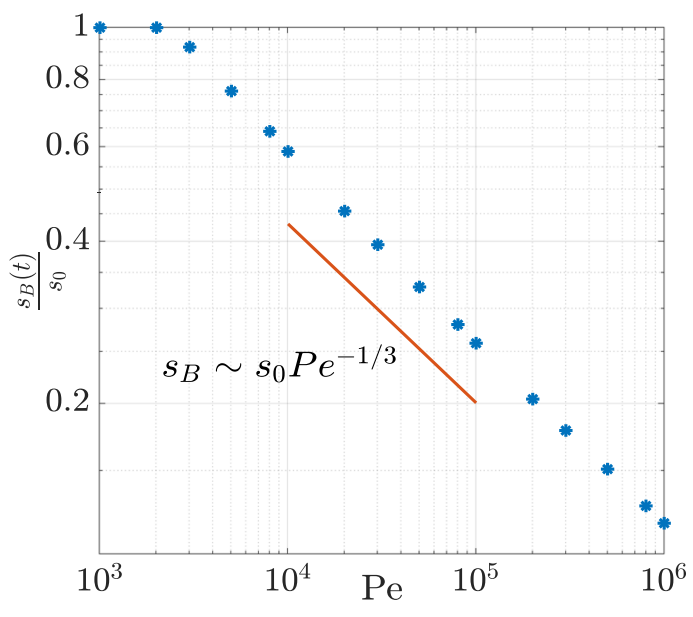

(c)

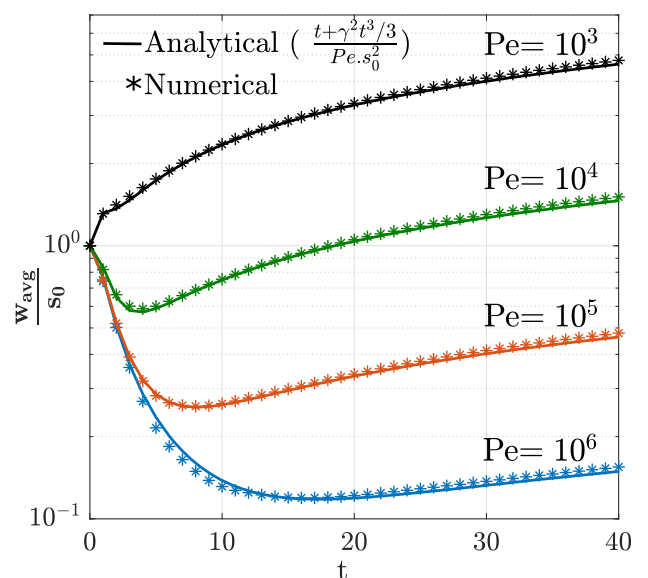

(b)

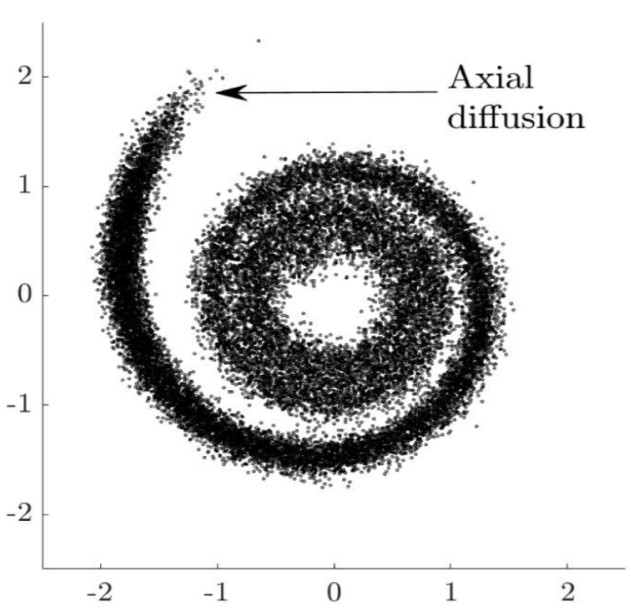

(d)

FIG. 6. (a) Shearing of a strip of scalar due to the presence of a velocity gradient in the direction perpendicular to the longest dimension of the strip. (b) Evolution of the strip width in time; here, $\gamma=\nabla v$. (c) The numerically obtained values for the Batchelor scale through the simulation (averaged minimum width of strips during the simulation) are represented by the marker $*$ and are plotted over a range of Péclet numbers. We observe a good agreement between the numerical data and the power law scaling between the Batchelor scale and Péclet number predicted by the theory. (d) Random walk particle tracking (RWPT) in a Rankine vortex for Pe $=10^{3}$. The reconstruction is equivalent to that of the Eulerian simulation, with the phenomenon of axial diffusion being observed here but not in the Lagrangian Ranz stretch model.

limits of the tracer, i.e., $0.45<r<2.0$. The plot shows an excellent agreement between analytical predictions and data from the numerical routine, thus, justifying our assumption that a single stretching rate governs the stirring protocol in the vortex flow. The power law scaling corresponding to the dependence of the Batchelor scale on Péclet numbers also matches well with numerical results [Fig. 6(c)]. The blue points correspond to the minimum width achieved in the simulation for a particular Péclet number. In Fig. 6(d), we show the scalar distribution in a Rankine vortex for $\mathrm{Pe}=10^{3}$, computed using a simple random walk particle tracking (RWPT) algorithm. The RWPT starts with an initial set of points distributed in space according to the initial condition $e^{-\tilde{n}^{2}}$. The advection step is carried out by solving $\frac{d \mathbf{x}_{\mathbf{i}}}{d t}=\mathbf{v}_{\mathbf{i}}\left(\mathbf{x}_{\mathbf{i}}\right)$ using an explicit Runge-Kutta method, and diffusion is accounted for by making the particles move an additional step given by $\sqrt{2 \Delta t / \mathrm{Pe}} \times$ randn, where $\Delta t=0.01$ is the time step used in the simulation and "randn" is an in-built MATLAB function that returns a scalar drawn from the standard normal distribution. This result is used for the purpose of verifying the concentration PDF in the coalescence regime, as described later in Sec. III C.

\section{B. Reconstruction of spatial distribution of scalar}

The task of reconstructing the spatial distribution of the scalar on a $2 \mathrm{D}$ grid is computationally very intensive. An alternative to a 


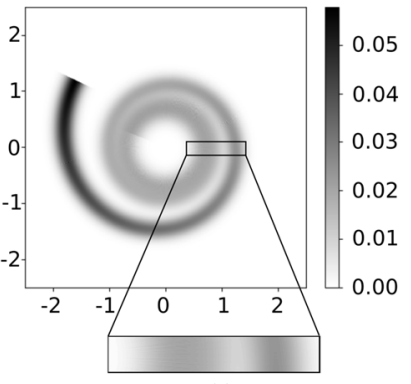

(a)

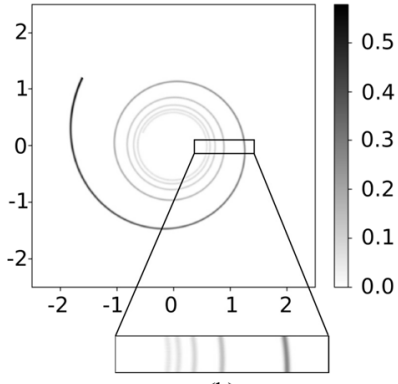

(b)
FIG. 7. Reconstruction of spatial distribution of scalar at $t=20$ for $\mathrm{Pe}=(\mathrm{a}) 10^{3}$ and (b) $10^{5}$. The vertical color bars beside the figures represent the normalized scalar concentration.

complete spatial reconstruction is to derive mixing characteristics from the evolution of $q_{\max }=1 / \sqrt{1+4 \tau_{i}(t)}$ and that of $\rho=\Delta x_{i} / \Delta x_{i}^{0}$.

However, this technique will be inaccurate in our case since it does not account for the significant overlap between different parts of the strip as it undergoes stretching and folding. Hence, a complete reconstruction becomes essential in order to capture coalescence. We reconstruct the spatial distribution of the scalar on a $2 \mathrm{D}$ grid $(-2.5 \leq x, y \leq 2.5)$ of dimension $1024 \times 1024$ for two representative Péclet numbers using the diffusive strip method as the much faster alternative to fully resolved Eulerian simulations. Figure 7 shows the numerical reconstruction of the spatial distribution of scalar at $\mathrm{t}=20$, where the time has been normalized by the time scale for advection $t_{a d v} \sim \mathcal{O}(1)$. In Fig. 7(a), corresponding to $\mathrm{Pe}=10^{3}$, the effect of stretching-enhanced diffusion can clearly be seen in many parts of the material lamella, especially at the region where the flow field changes its nature from linear to hyperbolic. Higher velocity gradients in that zone cause greater stretching and consequently more diffusion broadening. Different parts of the strip have overlapped to a significant extent over there, destroying concentration gradients, giving an indication that the mixing time had been attained well before. On the other hand, in Fig. 7(b), the concentration has not decayed much at parts of the strip away from the center of the vortex, whereas segments closer to the vortex eye have just started to overlap, implying that the mixing time has just been reached for $\mathrm{Pe}=10^{5}$.

\section{Probability density function of concentration}

An accurate description of the evolution of the probability density function of the scalar is an important component of understanding the mixing dynamics of a flow field. ${ }^{72,73}$ Numerically, the PDF can be obtained by computing the histogram of the spatial distribution of scalar over the $2 \mathrm{D}$ grid. This is computationally intensive since it requires a full reconstruction on a $2 \mathrm{D}$ grid. However, there is a faster alternative that uses the fact that each strip has a Gaussian transverse variation. This is done by computing the histogram of concentrations due to a solitary strip in the material line and then adding the contributions from each strip in order to get the total PDF. A linear sum computed over the strip will not yield the correct result once coalescence occurs; hence, this method is valid only at initial stages when different parts of the strip do not overlap. In spite of its limited accuracy, this is an important quantity as its deviation from the numerically computed PDF will be a measure of the extent of mixing that has occurred in the flow (see Appendix A for more details). Figure 8(a) shows the PDF of the concentration of scalar for $\mathrm{Pe}=10^{3}$ at $t=15$. Unlike the PDF for $\mathrm{Pe}=10^{5}$, we observe a much faster decay in concentration in this case since diffusion plays a more prominent role in low Péclet number flows. The numerically obtained PDF is seen to have a higher frequency of cells in intermediate and high concentration regimes than that in the PDF (in blue) obtained analytically by assuming that the strips constituting the material line do not overlap as they evolve. However, the aggregation of different parts of the scalar support is a reality when the strip width, defined by $w_{i}=s_{i} \sqrt{1+4 \tau_{i}}$, is of the order of the distance separating adjacent segments of the folded strip. This aggregation or

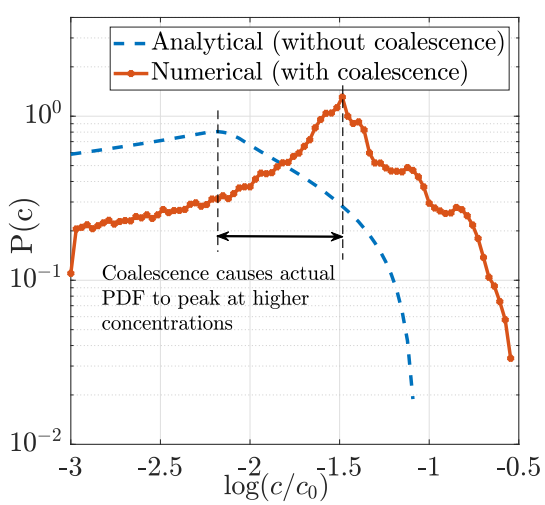

(a)

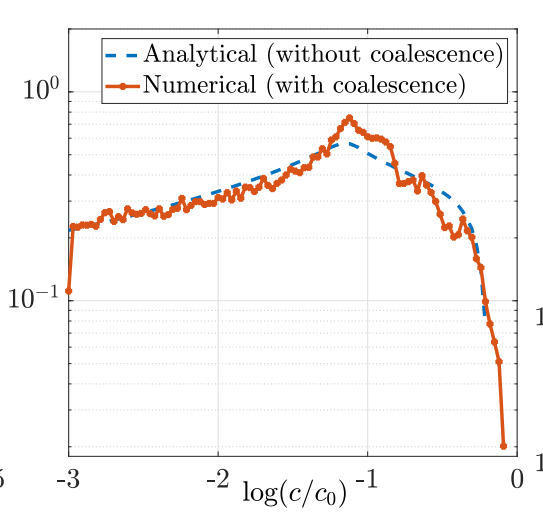

(b)

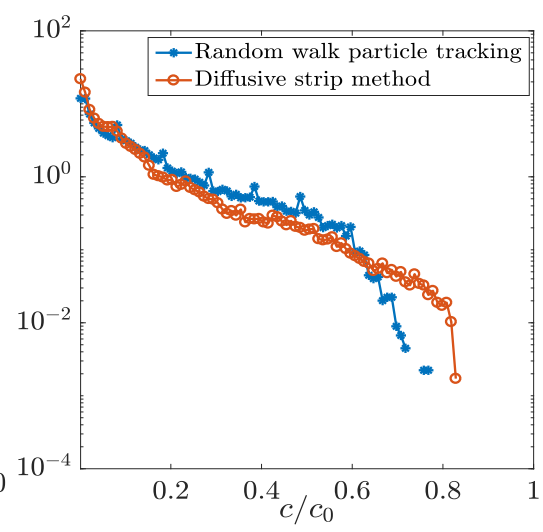

(c)

FIG. 8. PDF of concentration of scalar for (a) $P e=10^{3}$ and $(b) P e=10^{5}$ at $t=15$. (c) The PDF in the coalescence regime is validated with the PDF obtained from the random walk particle tracking simulation in a Rankine vortex. 
re-connection between different parts of the mixing interface causes the PDF to peak at higher concentrations. On the other hand, the two different versions of the PDF in Fig. 8(b) are a good match with one another because, at that stage of the simulation, the reconnection of lamellae had not occurred for $\mathrm{Pe}=10^{5}$. The PDF has also been computed using random walk particle tracking in a Rankine vortex flow field to validate the diffusive strip method [Fig. 8(c)]. The difference in the two arises due to axial diffusion, shown clearly in Fig. 6(d), a phenomenon captured in Eulerian simulations (see Fig. 2 also) but not in the Ranz stretch model.

It must be noted that the numerical PDF was obtained by assuming that the concentration at an arbitrary position in space is equal to a simple addition of the concentration contributions from different Gaussian segments. This is a valid construction rule for the PDF since the Fourier equation that governs the evolution of the tracer is linear, thereby allowing us to superimpose the concentration fields from various sources. This construction rule amounts to saying that if the concentration $c$ at a certain position in space is due to the addition from multiple (say, two), independent but identical sources, then the distribution $\mathrm{P}(\mathrm{c})$ of the concentration $\mathrm{c}$ in the resulting mixture is given by the self-convolution,

$$
P(c)=\int_{0}^{c} P_{1}\left(c_{1}\right) P_{2}\left(c-c_{1}\right) d c_{1},
$$

where $P_{1}$ and $P_{2}$ are identical. The situation that we are analyzing is that of an ever-dispersing mixture since the scalar does not have a compact support. For such a scenario, we observe the relaxation of the mixture toward a uniform state and bring out the role of the Péclet number in this process. Specifically, we compare the PDF in the coalescence regime, computed using the diffusive strip method, with an aggregation model in order to gain deeper insight into how the shape of the PDF evolves. ${ }^{75}$ In the PDF shown in Fig. 9(a), we observe a cusp near zero followed by an exponential decay. The existence of exponential tails is consistent with existing formulations on the evolution of the concentration PDF of a dye diluting in an unbounded medium. ${ }^{76,77}$ There is a steepening of the tail in time, and we observe a dependence of the kind $P(c) \sim \exp (-\alpha C), \alpha=t \cdot f(\mathrm{Pe})$, where $f(\mathrm{Pe})$ is observed to be a decreasing function of Péclet number. The physical significance of the rate of decay of the tail is that it is proportional to the amount of material overlap or equivalently proportional to the number of self-convolutions of individual concentration distributions.

We identify the onset of coalescence across a range of Péclet numbers by observing a characteristic time at which the analytical and numerical PDFs start deviating from each other. We introduce some notation that makes it easier to follow the results-let us assume that the numerical PDF peaks at $c=c_{1}$ and the analytical PDF peaks at $c=c_{2}$. On a logarithmic scale, the peaks are at $\log \left(c_{1}\right)$ and $\log \left(c_{2}\right)$, respectively. Let the difference between the peaks be denoted by $\Delta \log \left(c / c_{0}\right)=\log \left(c_{1}\right)-\log \left(c_{2}\right)$ such that larger values of $\Delta \log \left(c / c_{0}\right)$ indicate a greater separation between the peaks of the two PDFs, which in turn represents a greater degree of coalescence. The cross correlation function of the two PDFs is computed, and we observe the peak of this function shifting to higher values of $\Delta \log \left(c / c_{0}\right)$ with time. This shifting of the peak for lower Péclet is seen in Fig. 10, where the normalized cross correlation at two times (a) $t=7$ and (b) $t=50$, for $\mathrm{Pe}=10^{3}, 10^{5}$, and $10^{7}$ is shown, where the characteristic deviation time is defined as the time at which the peak of the cross correlation function crosses $\Delta \log \left(c / c_{0}\right)=k$. Here, $k$ can be set arbitrarily based on a threshold that the end user of this algorithm sets as a suitable indicator of coalescence.

\section{Impact of coalescence on reaction kinetics in a Rankine vortex}

We extend the ideas developed in Subsection II B to study the impact of coalescence on reaction kinetics during scalar mixing in a Rankine vortex. Using the numerical reconstruction of the spatial distribution of concentration, and by considering the same "fast," equilibrium-controlled bimolecular precipitation reaction that we analyzed in a linear shear flow, we compare the reactivity predicted by the independent lamellar model of mixing and that obtained

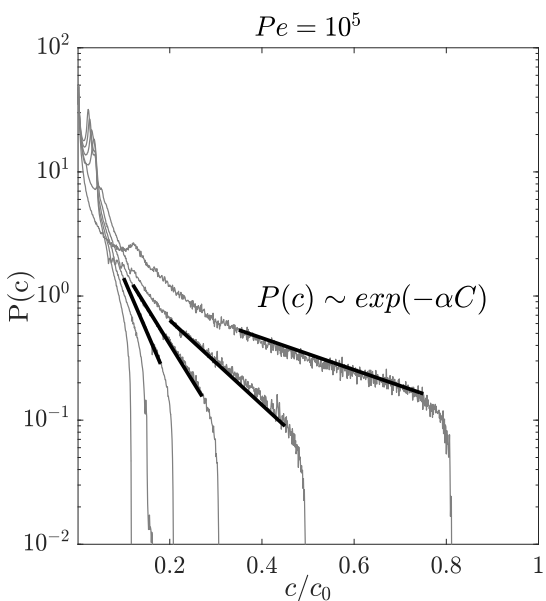

(a)

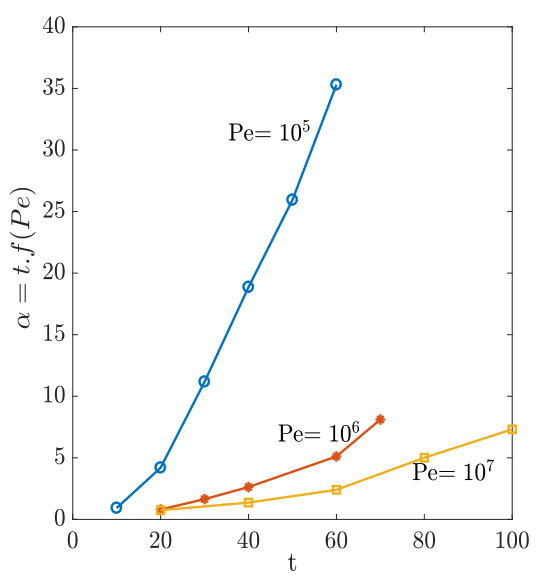

(b)
FIG. 9. (a) The PDF in the coalescence regime is obtained using the diffusive strip method. It is observed to have a cusp near 0 and an exponential falloff, consistent with formulations of the dilution of a passive scalar in an unbounded medium. (b) The slope of the exponential tail in the PDF at any given point in time is seen to be proportional to the number of reconnections of the mixing interface. 


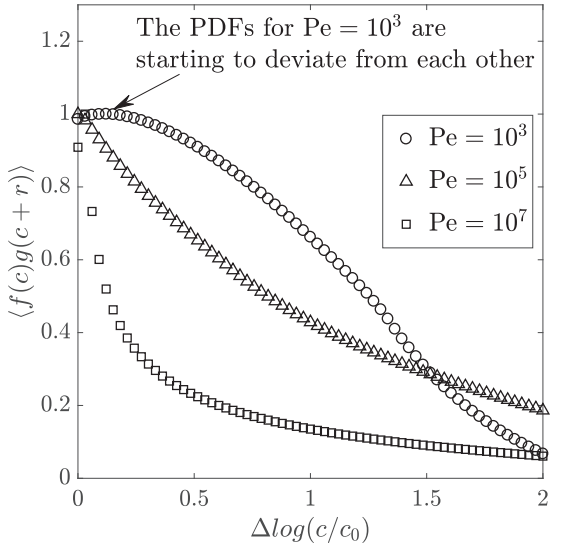

(a)

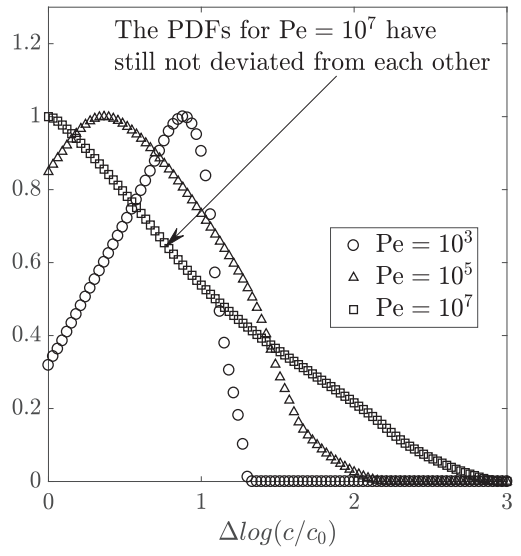

(b) in a coalescence regime. It has been shown for mixing-limited bimolecular reactions yielding a precipitate that the rate of reaction is proportional to the square of the spatial gradient of the concentration field [see Eq. (13)]. In a flow where we assume that the solute concentration evolves under an independent lamella model, that is, each strip evolves as if it is the solitary strip in the flow, the mixing rate is higher than that in an actual flow where the reconnection of different parts of the mixing interface is possible. In the latter, concentration gradients are smothered due to diffusive coalescence leading to material overlap, and in turn, the reactivity in the flow domain decreases.

These areas of coalesced scalar are characterized by the absence of distinguishable lamellae. On the other hand, zones where the lamellar structure of the scalar is preserved create steeper concentration gradients leading to faster reaction rates. These interfaces with sharp concentration gradients strongly influence subsurface dynamics since they act as hotspots for biochemical reactions. Such reactive hotspots create an environment for the development of microorganisms leading to the formation of biofilms, govern the reaction dynamics at the interface of saltwater and freshwater bodies, and also play a crucial role in chemical injection based remediation of contaminated groundwater. Since we are considering an ever-dispersing mixture, and not a confined mixture, the average concentration of the mixture will eventually homogenize to zero everywhere in the domain. However, the time it takes to do so will be different for different Péclet numbers.

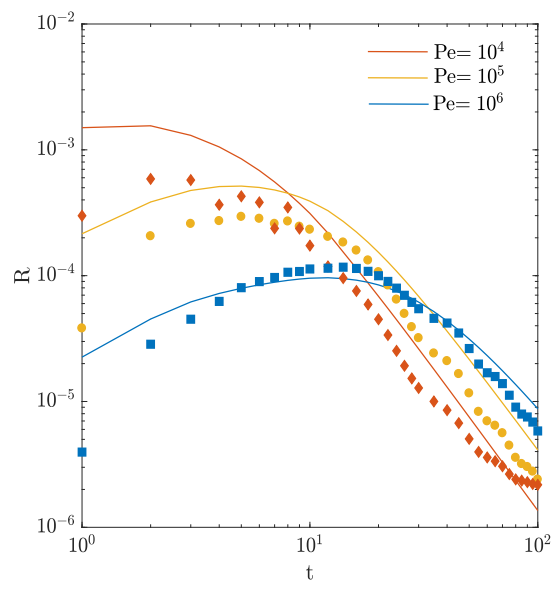

(a)

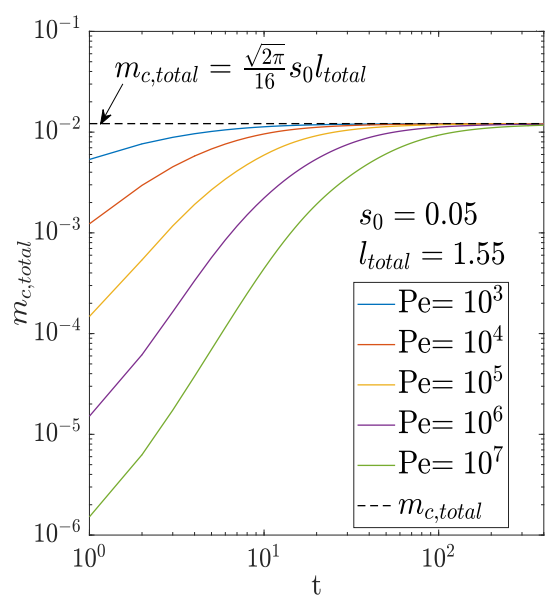

(b)

FIG. 11. (a) We highlight the effect of coalescence on reactivity in this plot. The integrated reactivity $R=\iint r d n d l$ from the independent lamella model (solid line) is compared to that obtained from the numerical reconstruction using the diffusive strip method (markers). The reactivity in the presence of coalescence or, equivalently, in the absence of discrete lamellar structures is seen to be less than that in the absence of coalescence. The onset of coalescence smothers concentration gradients, and this in turn reduces the reactivity. Thus, the role of coalescence is seen to be that of decreasing the rate of product formation. (b) The mass of precipitate, calculated using the independent lamella model, is plotted against time for a range of Péclet numbers. In both figures, the results are shown for $K_{e q}=1$. 
The time evolution of the reactivity integrated over the spatial coordinates yields a trend that is consistent with the hypothesis that the presence of lamellar structures, or equivalently higher concentration gradients, enhances reaction rates. The predictions for the integrated reactivity [Eq. (17)] in the independent lamella model (which does not account for coalescence) have been shown to slightly exceed those obtained from a high resolution reconstruction using the diffusive strip method (which accounts for coalescence) in Fig. 11(a). These results clearly indicate that the discerning feature governing reaction kinetics at the interface is the extent of coalescence or its absence thereof. This insight is crucial in understanding how much mass is produced in a given span of time for a precipitation/dissolution reaction driven by mixing. Just as in the case of a linear shear flow, we observe that after a sufficient time, all the mass in the lamella is consumed to yield the equilibrium precipitate mass, and in the limit $t \gg t_{\text {mix }}$, the total mass formed in the domain equals $m_{c, \text { total }}=\left(s_{0} l_{\text {total }} / 16\right) \sqrt{\frac{2 \pi}{K_{e q}}}$, where $l_{\text {total }}$ is the initial length of the entire material line composed of multiple strips [see Fig. 11(b)]. The similarity in the mass dynamics for a linear shear and Rankine vortex can be attributed to the fact established in Sec. III A that the substrate deformation dynamics in a Rankine vortex is essentially the same as that in a linear shear where material lines elongate linearly in time.

It is important to note that at very long times, the same mass of product is formed irrespective of the model (coalescence vs no coalescence) since we have the same mass of reactants to start with in both models. Hence, the role of coalescence is to reduce concentration gradients only until a certain stage in the stirring protocol, and by that point in time, much of the reactants have been converted to the product. Therefore, the difference between the models lies in the rate at which the final mass asymptote [Eq. (15)] is reached, the rate being faster in the independent lamellar model, since the reactivity is higher there in the initial stages by virtue of larger concentration gradients. Thus, understanding the mixing and reaction dynamics in such prototypical laminar flows is the first step toward a mechanistic understanding of transport in reactive flow systems. The ubiquity of the Rankine vortex makes this fundamental study an important contribution to our understanding of mixing-limited reactive transport.

\section{CONCLUSION}

In this work, we have demonstrated the influence of stretching on mixing and reaction kinetics for mixing-limited reactions in two prototypical flow models, the linear shear and Rankine vortex. The Ranz stretch model has been used to reconstruct the distribution of a conservative scalar in two dimensions, and a key signature of coalescence during scalar mixing has been identified as the difference between the actual PDF of concentration, obtained from numerical simulations by exploiting the linear nature of the Fourier equation, and the concentration PDF obtained from analytical considerations, which does not account for the phenomena of strip overlap.

We have then established the fundamental behavior of species undergoing an equilibrium-controlled precipitation reaction. The lamellar representation of mixing has been used to decouple the advection process from the diffusion-reaction processes, and using this model, we derive closed-form analytical expressions for the local reactivity, upscaled reactivity, and mass of precipitate obtained at any given point in time as a function of transport and reaction parameters. The role of fluid deformation due to the inherent heterogeneity in the flow is shown to significantly enhance reactivity than that for a homogeneous flow. Concentration gradients and reactivity are observed to increase until the Péclet number dependent mixing time, beyond which gradients are smothered and a simultaneous decay in the reactivity occurs. Importantly, the mass of precipitate after sufficient time is shown to be independent of the Péclet number, and the role of the Péclet number is seen to be important only at $t<t_{m i x}$ for the mass dynamics. Finally, we have highlighted the impact of coalescence during scalar transport in a Rankine vortex on reaction kinetics where the smothering of concentration gradients due to the onset of coalescence is shown to decrease the upscaled reactivity in comparison to the integrated reactivity obtained from an independent lamella model.

We hope that this work improves our fundamental understanding of scalar dispersion in a vortex and that of mixing-driven reactive flows. These ideas may be very useful if extended to simulations of three-dimensional flows in porous media where scalar blobs are sheared at different rates and will lay the foundation for studying reactive mixing in groundwater flows.

\section{ACKNOWLEDGMENTS}

The authors would like to thank the H2020 European Research Council, ReactiveFronts No. 648377, for financial support.

\section{APPENDIX A: ANALYTICAL EXPRESSION FOR CONCENTRATION PDF IN AN INDEPENDENT LAMELLA MODEL}

We derive the analytical expression for the PDF of concentration $P(c)$ by making the following change of variables:

$$
P(c ; t) d c=Q(x ; t) d x,
$$

where the concentration PDF $P(c ; t)$ is defined so that $P(c ; t) d c$ is the probability, or frequency of occurrence, of concentration $c$ at time $t$ in the domain and $Q(x ; t) d x$ is the mass in an area $d x$ centered at $x$. Here, we assume a uniform distribution of $x$ over a fixed domain of interest. This allows us to write

$$
P(c)=\frac{Q(x)}{\left|\frac{d c}{d x}\right|} .
$$

At an arbitrary time in the simulation, the concentration for a solitary strip in the flow is given by Eq. (9). Substituting Eq. (9) in (A2) yields

$$
\frac{d c}{d x}=c \frac{-2 x}{s^{2}(1+4 \tau)}
$$

with $x \equiv n$. Separating the variables and integrating from the strip center to a certain distance normal to the strip in the local coordinate system, we get

$$
\int_{c_{\max }}^{c} \frac{1}{c^{\prime}} d c^{\prime}=\int_{0}^{x} \frac{-2 x^{\prime}}{s^{2}(1+4 \tau)} d x^{\prime}
$$


where $c_{\max }=1 / \sqrt{(1+4 \tau)}$. The integration above gives an expression for $\mathrm{x}$, which when substituted in (A3) gives the final expression for the PDF for the $i$ th segment as

$$
P_{i}(c)=A \frac{s_{i} \Delta x_{i} \sqrt{1+4 \tau_{i}}}{c \sqrt{\ln \left(c_{\max , i} / c\right)}}
$$

where $c_{\text {max }, i}=1 / \sqrt{\left(1+4 \tau_{i}\right)}$. The normalizing constant, A, cannot be obtained from this analytical expression since the integral $\int_{-\infty}^{\infty} P(c) d c=1$ is divergent at $c=0$. The global PDF is calculated by adding the contributions from all strips, assuming they do not overlap with each other.

\section{APPENDIX B: DETAILS OF EULERIAN SIMULATIONS}

The task is to solve the following equation in the Eulerian framework:

$$
\frac{\partial c}{\partial t}+\mathbf{v} \cdot \nabla c=D \nabla^{2} c
$$

We solve the advection-diffusion equation using in-built solvers in Basilisk. $^{78}$ The advection equation $\frac{\partial c}{\partial t}+\mathbf{u} \cdot \nabla c=0$ is solved using the Bell-Collela-Glaz (BCG) scheme that is a flux-based advection scheme having second-order accuracy in time. While computing the gradient of the scalar, instead of a cell-centered gradient, a generalized "minmod" limiter is used where the parameter $\theta$, which decides the limiting scheme, is set to 1.3 to prevent spurious oscillations in the scalar field. ${ }^{79}$ The diffusion equation $\frac{\partial c}{\partial t}=\nabla \cdot(D \nabla c)$, on the other hand, is solved using the diffusion solver in Basilisk. Finally, an adaptive grid, where the two-dimensional space is partitioned using a quadtree data structure, is implemented at each time step to reduce computation time and errors due to numerical diffusion. ${ }^{80}$ The grid used for reconstruction of the scalar had the same limits as that used in the diffusive strip method (refer to Sec. III B) and was of dimension $1024 \times 1024$ with a maximum and minimum of 12 and 10 levels of refinement, respectively.

\section{DATA AVAILABILITY}

The data that support the findings of this study are available from the corresponding author upon reasonable request.

\section{REFERENCES}

${ }^{1}$ C. Zheng and G. D. Bennett, Applied Contaminant Transport Modeling (WileyInterscience, New York, 2002), Vol. 2.

${ }^{2}$ C. K. Ho, L. Orear, Jr., J. L. Wright, and S. A. McKenna, Water Distribution Systems Analysis Symposium 2006 (ASCE, 2008), pp. 1-18.

${ }^{3} \mathrm{~K}$. Caldeira and G. H. Rau, "Accelerating carbonate dissolution to sequester carbon dioxide in the ocean: Geochemical implications," Geophys. Res. Lett. 27, 225, https://doi.org/10.1029/1999gl002364 (2000).

${ }^{4}$ C. Brekke and A. H. S. Solberg, "Oil spill detection by satellite remote sensing," Remote Sens. Environ. 95, 1 (2005).

${ }^{5}$ Y. Liu, R. H. Weisberg, C. Hu, and L. Zheng, "Tracking the deepwater horizon oil spill: A modeling perspective,” EOS, Trans. Am. Geophys. Union 92, 45 (2011).

${ }^{6}$ T. Tél, A. de Moura, C. Grebogi, and G. Károlyi, "Chemical and biological activity in open flows: A dynamical system approach," Phys. Rep. 413, 91 (2005).
${ }^{7}$ L. Burté, C. A. Cravotta III, L. Bethencourt, J. Farasin, M. Pédrot, A. Dufresne, M.-F. Gérard, C. Baranger, T. Le Borgne, and L. Aquilina, "Kinetic study on clogging of a geothermal pumping well triggered by mixing-induced biogeochemical reactions," Environ. Sci. Technol. 53, 5848 (2019).

${ }^{8}$ W. D. Mohr, R. L. Saxton, and C. H. Jepson, "Mixing in laminar-flow systems," Ind. Eng. Chem. 49, 1855 (1957).

${ }^{9}$ A. W. Nienow, M. F. Edwards, and N. Harnby, Mixing in the Process Industries (Butterworth-Heinemann, 1997).

${ }^{10}$ S. Cerbelli, M. M. Alvarez, and F. J. Muzzio, "Prediction and quantification of micromixing intensities in laminar flows," AIChE J. 48, 686 (2002).

${ }^{11}$ O. Levenspiel and K. B. Bischoff, Advances in Chemical Engineering (Elsevier, 1964), Vol. 4, pp. 95-198.

${ }^{12}$ L. F. Konikow and D. B. Grove, "Derivation of equations describing solute transport in ground water," Technical Report No. 77-19, USGS, 1977.

${ }^{13} \mathrm{~J}$. Bear, Dynamics of Fluids in Porous Media (Courier Corporation, 2013).

${ }^{14} \mathrm{~J}$. D. Bredehoeft and G. F. Pinder, "Mass transport in flowing groundwater," Water Resour. Res. 9, 194, https://doi.org/10.1029/wr009i001p00194 (1973).

${ }^{15} \mathrm{~S}$. Whitaker et al., "The transport equations for multi-phase systems," Chem. Eng. Sci. 28, 139 (1973).

${ }^{16}$ L. F. Konikow and J. D. Bredehoeft, Computer Model of Two-Dimensional Solute Transport and Dispersion in Ground Water (US Government Printing Office, Washington, DC, 1978), Vol. 7.

${ }^{17}$ G. F. Pinder and H. H. Cooper, Jr., "A numerical technique for calculating the transient position of the saltwater front," Water Resour. Res. 6, 875, https://doi.org/10.1029/wr006i003p00875 (1970).

${ }^{18}$ A. O. Garder, Jr., D. W. Peaceman, A. L. Pozzi, Jr. et al., "Numerical calculation of multidimensional miscible displacement by the method of characteristics," Soc, Pet. Eng. J. 4, 26 (1964).

${ }^{19}$ D. L. Reddell and D. K. Sunada, Hydrology Papers (Colorado State University, 1970), p. 41.

${ }^{20} \mathrm{~J}$. Rubin, "Transport of reacting solutes in porous media: Relation between mathematical nature of problem formulation and chemical nature of reactions," Water Resour. Res. 19, 1231, https://doi.org/10.1029/wr019i005p01231 (1983).

${ }^{21}$ J. Rubin, "Solute transport with multi-segment, equilibrium-controlled reactions: A feed-forward simulation method," Water Resour. Res. 26, 2029, https://doi.org/10.1029/90wr00773 (1990).

${ }^{22} \mathrm{~J}$. Rubin, "Solute transport with multisegment, equilibrium-controlled, classical reactions: Problem solvability and feed forward method's applicability for complex segments of at most binary participants," Water Resour. Res. 28, 1681, https://doi.org/10.1029/92wr00572 (1992).

${ }^{23}$ J. C. Friedly and J. Rubin, "Solute transport with multiple equilibriumcontrolled or kinetically controlled chemical reactions," Water Resour. Res. 28, 1935, https://doi.org/10.1029/92wr00699 (1992).

${ }^{24} \mathrm{M}$. W. Saaltink, J. Carrera, and C. Ayora, "On the behavior of approaches to simulate reactive transport,” J. Contam. Hydrol. 48, 213 (2001).

${ }^{25} \mathrm{M}$. W. Saaltink, C. Ayora, and J. Carrera, "A mathematical formulation for reactive transport that eliminates mineral concentrations," Water Resour. Res. 34, 1649, https://doi.org/10.1029/98wr00552 (1998).

${ }^{26}$ S. Molins, J. Carrera, C. Ayora, and M. W. Saaltink, "A formulation for decoupling components in reactive transport problems," Water Resour. Res. 40, W10301, https://doi.org/10.1029/2003wr002970 (2004).

${ }^{27}$ M. De Simoni, J. Carrera, X. Sánchez-Vila, and A. Guadagnini, "A procedure for the solution of multicomponent reactive transport problems," Water Resour. Res. 41, W11410, https://doi.org/10.1029/2005wr004056 (2005).

${ }^{28}$ M. De Simoni, X. Sanchez-Vila, J. Carrera, and M. W. Saaltink, "A mixing ratios-based formulation for multicomponent reactive transport," Water Resour. Res. 43, W07419, https://doi.org/10.1029/2006wr005256 (2007).

${ }^{29}$ R. K. Ballentine, S. R. Reznek, and C. W. Hall, Subsurface Pollution Problems in the United States (US Environmental Protection Agency, Office of Water Programs, Applied Technology Division, 1972).

${ }^{30}$ M. P. Anderson and J. A. Cherry, "Using models to simulate the movement of contaminants through groundwater flow systems," Crit. Rev. Environ. Sci. Technol. 9, 97 (1979). 
${ }^{31}$ J. F. Pickens and W. C. Lennox, "Numerical simulation of waste movement in steady groundwater flow systems," Water Resour. Res. 12, 171, https://doi.org/10.1029/wr012i002p00171 (1976).

${ }^{32}$ T. R. Blake and S. K. Garg, "On the species transport equation for flow in porous media," Water Resour. Res. 12, 748, https://doi.org/10.1029/wr012i004p00748 (1976).

${ }^{33}$ M. Rezaei, E. Sanz, E. Raeisi, C. Ayora, E. Vázquez-Suné, and J. Carrera, "Reactive transport modeling of calcite dissolution in the fresh-salt water mixing zone," J. Hydrol. 311, 282 (2005)

${ }^{34} \mathrm{~S}$. Emmanuel and B. Berkowitz, "Mixing-induced precipitation and porosity evolution in porous media," Adv. Water Resour. 28, 337 (2005).

${ }^{35}$ L. S. Balistrieri, S. E. Box, and J. W. Tonkin, "Modeling precipitation and sorption of elements during mixing of river water and porewater in the Coeur d'Alene River Basin,” Environ. Sci. Technol. 37, 4694 (2003).

${ }^{36}$ M. Dentz and J. Carrera, "Mixing and spreading in stratified flow," Phys. Fluids 19, 017107 (2007).

${ }^{37}$ D. Griffani, P. Rognon, B. Metzger, and I. Einav, "How rotational vortices enhance transfers," Phys. Fluids 25, 093301 (2013).

${ }^{38}$ T. L. Borgne, T. R. Ginn, and M. Dentz, "Impact of fluid deformation on mixinginduced chemical reactions in heterogeneous flows," Geophys. Res. Lett. 41, 7898, https://doi.org/10.1002/2014gl062038 (2014).

${ }^{39}$ P. De Anna, M. Dentz, A. Tartakovsky, and T. Le Borgne, "The filamentary structure of mixing fronts and its control on reaction kinetics in porous media flows," Geophys. Res. Lett. 41, 4586, https://doi.org/10.1002/2014gl060068 (2014).

${ }^{40}$ A. J. Valocchi, D. Bolster, and C. J. Werth, "Mixing-limited reactions in porous media," Transp. Porous Media 130, 157 (2019).

${ }^{41}$ K. R. Pratt, J. D. Meiss, and J. P. Crimaldi, "Reaction enhancement of initially distant scalars by Lagrangian coherent structures," Phys. Fluids 27, 035106 (2015).

${ }^{42} \mathrm{~N}$. Cagney and S. Balabani, "Lagrangian structures and mixing in the wake of a streamwise oscillating cylinder," Phys. Fluids 28, 045107 (2016).

${ }^{43}$ T. Le Borgne, P. D. Huck, M. Dentz, and E. Villermaux, "Scalar gradients in stirred mixtures and the deconstruction of random fields," J. Fluid Mech. 812, 578 (2017).

${ }^{44}$ T. Le Borgne, M. Dentz, and E. Villermaux, "The lamellar description of mixing in porous media," J. Fluid Mech. 770, 458 (2015).

${ }^{45}$ D. R. Lester, M. Dentz, and T. Le Borgne, "Chaotic mixing in three-dimensional porous media," J. Fluid Mech. 803, 144 (2016).

${ }^{46}$ R. Turuban, D. R. Lester, T. Le Borgne, and Y. Méheust, "Space-group symmetries generate chaotic fluid advection in crystalline granular media," Phys. Rev. Lett. 120, 024501 (2018).

${ }^{47}$ R. Turuban, D. R. Lester, J. Heyman, T. L. Borgne, and Y. Méheust, "Chaotic mixing in crystalline granular media," J. Fluid Mech. 871, 562 (2019).

${ }^{48}$ E. Villermaux, "Mixing versus stirring," Annu. Rev. Fluid Mech. 51, 245 (2019).

${ }^{49}$ J. Heyman, D. R. Lester, R. Turuban, Y. Méheust, and T. Le Borgne, "Stretching and folding sustain microscale chemical gradients in porous media," Proc. Natl. Acad. Sci. U. S. A. 117, 13359 (2020).

${ }^{50}$ T. R. Meyer, J. C. Dutton, and R. P. Lucht, "Vortex interaction and mixing in a driven gaseous axisymmetric jet," Phys. Fluids 11, 3401 (1999).

${ }^{51}$ J. P. Crimaldi, J. R. Cadwell, and J. B. Weiss, "Reaction enhancement of isolated scalars by vortex stirring," Phys. Fluids 20, 073605 (2008).

${ }^{52}$ J. J. Hidalgo, M. Dentz, Y. Cabeza, and J. Carrera, "Dissolution patterns and mixing dynamics in unstable reactive flow," Geophys. Res. Lett. 42, 6357, https://doi.org/10.1002/2015gl065036 (2015).

${ }^{53}$ L. Bates, D. Bradley, G. Paczko, and N. Peters, "Engine hot spots: Modes of autoignition and reaction propagation," Combust. Flame 166, 80 (2016).

${ }^{54} \mathrm{M}$. Rolle and T. Le Borgne, "Mixing and reactive fronts in the subsurface," Rev. Mineral. Geochem. 85, 111 (2019).

${ }^{55}$ W. E. Ranz, "Applications of a stretch model to mixing, diffusion, and reaction in laminar and turbulent flows," AIChE J. 25, 41 (1979).

${ }^{56} \mathrm{P}$. Meunier and E. Villermaux, "The diffusive strip method for scalar mixing in two dimensions," J. Fluid Mech. 662, 134 (2010).
${ }^{57}$ A. Poisson and A. Papaud, "Diffusion coefficients of major ions in seawater," Mar. Chem. 13, 265 (1983).

${ }^{58} \mathrm{R}$. E. Zeebe, "On the molecular diffusion coefficients of dissolved $\mathrm{CO}_{2}, \mathrm{HCO}_{3}{ }^{-}$ and $\mathrm{CO}_{3}{ }^{2-}$ and their dependence on isotopic mass," Geochim. Cosmochim. Acta 75, 2483 (2011)

${ }^{59}$ C. Steefel, D. DePaolo, and P. Lichtner, "Reactive transport modeling: An essential tool and a new research approach for the Earth sciences," Earth Planet. Sci. Lett. 240, 539 (2005).

${ }^{60}$ C. I. Steefel, C. A. J. Appelo, B. Arora, D. Jacques, T. Kalbacher, O. Kolditz, V. Lagneau, P. C. Lichtner, K. U. Mayer, J. C. L. Meeussen et al., "Reactive transport codes for subsurface environmental simulation," Comput. Geosci. 19, 445 (2015).

${ }^{61}$ P. Meunier and E. Villermaux, "How vortices mix," J. Fluid Mech. 476, 213 (2003).

${ }^{62} \mathrm{~J}$. Duplat and E. Villermaux, "Mixing by random stirring in confined mixtures," J. Fluid Mech. 617, 51 (2008).

${ }^{63}$ T. Le Borgne, M. Dentz, and E. Villermaux, "Stretching, coalescence, and mixing in porous media," Phys. Rev. Lett. 110, 204501 (2013).

${ }^{64}$ P. K. Kitanidis, "The concept of the dilution index," Water Resour. Res. 30, 2011, https://doi.org/10.1029/94wr00762 (1994).

${ }^{65}$ G. K. Batchelor, The Theory of Homogeneous Turbulence (Cambridge University Press, 1953).

${ }^{66}$ H. B. Bluestein, W.-C. Lee, M. Bell, C. C. Weiss, and A. L. Pazmany, "Mobile Doppler radar observations of a tornado in a supercell near bassett, Nebraska, on 5 June 1999. Part II: Tornado-vortex structure," Mon. Weather Rev. 131, 2968 (2003).

${ }^{67}$ R. A. Brown, B. A. Flickinger, E. Forren, D. M. Schultz, D. Sirmans, P. L. Spencer, V. T. Wood, and C. L. Ziegler, "Improved detection of severe storms using experimental fine-resolution WSR-88d measurements," Weather Forecast. 20, 3 (2005).

${ }^{68}$ M. Bertato, D. B. Giaiotti, A. Manzato, and F. Stel, "An interesting case of tornado in Friuli-Northeastern Italy," Atmos. Res. 67-68, 3 (2003).

${ }^{69} \mathrm{P}$. Magnico, "Hydrodynamic and transport properties of packed beds in small tube-to-sphere diameter ratio: Pore scale simulation using an Eulerian and a Lagrangian approach," Chem. Eng. Sci. 58, 5005 (2003).

${ }^{70}$ M. Aminpour, S. A. Galindo-Torres, A. Scheuermann, and L. Li, "Pore-scale behavior of Darcy flow in static and dynamic porous media," Phys. Rev. Appl. 9, 064025 (2018).

${ }^{71}$ G. K. Batchelor, "Small-scale variation of convected quantities like temperature in turbulent fluid Part 1. General discussion and the case of small conductivity," J. Fluid Mech. 5, 113 (1959).

${ }^{72} \mathrm{P}$. Meunier and E. Villermaux, "Van Hove singularities in probability density functions of scalars," C. R. Mec. 335, 162 (2007).

${ }^{73}$ A. Figueroa, P. Meunier, S. Cuevas, E. Villermaux, and E. Ramos, "Chaotic advection at large Péclet number: Electromagnetically driven experiments, numerical simulations, and theoretical predictions," Phys. Fluids 26, 013601 (2014).

${ }^{74}$ E. Villermaux and J. Duplat, "Mixing is an aggregation process," C. R. Mec. 331, 515 (2003).

${ }^{75}$ E. Villermaux, IUTAM Symposium on Turbulent Mixing and Combustion (Springer, 2002), pp. 1-21.

${ }^{76} \mathrm{~J}$. Sukhatme and R. T. Pierrehumbert, "Decay of passive scalars under the action of single scale smooth velocity fields in bounded two-dimensional domains: From non-self-similar probability distribution functions to self-similar eigenmodes," Phys. Rev. E 66, 056302 (2002).

${ }^{77}$ B. I. Shraiman and E. D. Siggia, "Lagrangian path integrals and fluctuations in random flow," Phys. Rev. E 49, 2912 (1994).

${ }^{78}$ S. Popinet, Basilisk, http://basilisk.fr/ (accessed 15 July 2019).

${ }^{79}$ A. Kurganov and E. Tadmor, "New high-resolution central schemes for nonlinear conservation laws and convection-diffusion equations," J. Comput. Phys. 160, 241 (2000).

${ }^{80}$ F. Pascal and J. Marechal, in Proceedings of the 7th International Meshing Roundtable, 1998 